\title{
Two Frequenins in Drosophila: unveiling the evolutionary history of an unusual Neuronal Calcium Sensor (NCS) duplication
}

\author{
Alejandro Sánchez-Gracia ${ }^{1,2^{*}}$, Jesús Romero-Pozuelo ${ }^{1,3}$, Alberto Ferrús ${ }^{1}$
}

\begin{abstract}
Background: Drosophila Frequenin (Frq), the homolog of the mammalian Neuronal Calcium Sensor-1 (NCS-1), is a high affinity calcium-binding protein with ubiquitous expression in the nervous system. This protein has an important role in the regulation of neurotransmitter release per synapse, axonal growth and bouton formation. In D. melanogaster, Frequenin is encoded by two genes (fral and fra2), a very unexpected feature in the Frq/NCS-1 subfamily. These genes are located in tandem in the same genomic region, and their products are 95\% identical in their amino acid sequence, clearly indicating their recent origin by gene duplication. Here, we have investigated the factors involved in this unusual feature by examining the molecular evolution of the two fra genes in Drosophila and the evolutionary dynamics of NCS family in a large set of bilaterian species.

Results: Surprisingly, we have found no amino acid replacements fixed across the twelve Drosophila species surveyed. In contrast, synonymous substitutions have been prevalent in the evolution of the coding region of fra 1 and fra2, indicating the presence of strong functional constraints following gene duplication. Despite that, we have detected that significant evolutionary rate acceleration had occurred in Frq1 in early times from the duplication, in which positive selection (likely promoting functional diversification) had probably an important role. The analysis of sequence conservation and DNA topology at the non-coding regions of both genes has allowed the identification of DNA regions candidates to be cis-regulatory elements. The results reveal a possible mechanism of regulatory diversification between fra 1 and fra2.

Conclusions: The presence of two Frequenins in Drosophila and the rapid accumulation of amino acid substitutions after gene duplication are very unusual features in the evolution of the Frq/NCS-1 subfamily. Here we show that the action of positive selection in concordance with some extent of regulatory diversification might explain these findings. Selected amino acid substitutions in Frq1 likely contributed to the functional divergence between the two duplicates, which, in turn, should have diverged in their regulation by Ecdysone-induced early genes.
\end{abstract}

\section{Background}

Many different aspects of neuronal function, including neurotransmitter release from synaptic vesicles, are regulated by alterations in the concentration of intracellular free $\mathrm{Ca}^{2+}[1,2]$. The transduction of calcium signals into appropriate physiological responses is frequently mediated by a range of calcium sensor proteins, which act as effectors and modulators in signalling pathways [3]. The specific effects of these changes depend on their affinity for

\footnotetext{
* Correspondence: alejandro.sanchez@ibe.upf-csic.es

${ }^{1}$ Instituto Cajal, CSIC, Ave. Dr. Arce 37, Madrid 28002, Spain
}

$\mathrm{Ca}^{2+}$, their cellular localization in relation to the $\mathrm{Ca}^{2+}$ entry signal and their interaction with other proteins. A number of calcium binding proteins related to the ubiquitous protein Calmodulin are overrepresented in or expressed only in the nervous system. These include the family of intracellular Neuronal Calcium Sensor (NCS) proteins [4-6]. Members of this protein family, which have been identified in many organisms ranging from yeast to mammals, bind $\mathrm{Ca}^{2+}$ at their EF-hand domains and regulate many important processes in neuronal signalling. This family can be divided into five subfamilies or classes (A-E) based on their amino acid sequence similarity [3]. 
Drosophila Frequenin (Frq), the homolog of the mammalian Neuronal Calcium Sensor-1 (NCS-1), was the founder member of the NCS family and the first to be characterized being, at this time, the only component of the class A [7]. Frequenin is a high affinity calciumbinding protein composed by two pairs of EF-hand motifs with ubiquitous expression in the nervous system. Its over-expression enhances facilitation and increases release per synapse $[8,9]$. Also, the levels of Frq in motoneuron control the number of boutons and branching, strongly suggesting a role in modulating axonal growth and bouton formation [8,9]. In Drosophila melanogaster, this calcium sensor is encoded by two different genes, frq 1 and frq 2 [9], which are located in tandem in the same genomic region (spaced by $11.6 \mathrm{~kb}$ ). These two genes show a similar intron-exon structure and a high amino acid sequence similarity (they differ in only 10 residues [9]). All these features clearly support an origin by gene duplication and suggest that the encoded proteins would still perform similar functions in D. melanogaster. In addition, the prospective search [7] across some fully sequenced genomes failed to detect duplicated copies belonging to this subfamily, with the exception of the zebrafish Danio rerio [10]. In this species, like in Drosophila, there are two independent Frequenin encoding genes. In $D$. melanogaster, both genes have very similar temporal and qualitative expression profiles but their quantitative levels differ [9]: while frq1 mRNA is 2-3 fold more abundant at the end of the embryo stage and in 1st instar larvae than in the adult, frq2 mRNA always reaches its peak expression level in the adult. Comparing the mRNA expression in the adult, frq1 shows a $20-30 \%$ higher level than frq2. Significant expression in the ventral ganglia was found for both genes, and this expression appears to be specific. Interestingly, the reduction or abolishment of Frequenin activity in flies does produce neither lethality nor sterility, which originally suggested a small contribution to individual fitness of these proteins. The analyses of over-expression and loss-of-function phenotypes, nevertheless, showed that mutants had larval locomotion defects, deficient synaptic transmission, impaired $\mathrm{Ca}^{2+}$ entry and enhanced nerve terminal growth, demonstrating a relevant role of Frequenin in the development and function of the nervous system $[9,11]$.

In order to understand the causes of the atypical preservation of two frq genes in Drosophila, we have analyzed nucleotide and amino acid sequence variation of frq 1 and frq 2 across the twelve species of the Drosophila genus with the complete genome sequenced [12]. Besides, we have also examined amino acid sequence evolution of the other members of NCS protein family to identify functionally important sites responsible for differentiation between subfamilies. We have found that positive selection had an important role in the fixation of some of the amino acid changes found between Drosophila Frequenins. The results also show that these changes probably shaped the functional differentiation between the two copies. Finally, the evolutionary analysis of non-coding sequences suggests that gene expression differences observed between these two genes might be, at least in part, consequence of the different evolution of regulatory regions after gene duplication.

\section{Results}

Two old and highly constrained Frequenins in Drosophila

We have found that the 12 Drosophila species have two genes encoding Frequenin. In all species, the orthologues of $f r q 1$ and $f r q 2$ are located in the syntenic region of the X chromosome and maintain nearly the same gene structure (i.e. exon/intron number and sizes; Figure 1 ). The frq1 genes of D. sechellia and D. pseudoobscura are situated in genomic contigs that contain several gaps filled with ' $N$ ' and they are not complete. In spite of that, partial coding regions available for these genes have no mutations truncating the open reading frames or generating stop codons, suggesting that both are functional copies. In order to be conservative in our further analysis, however, we used only complete coding regions for comparisons.

Both genes show high levels of silent nucleotide divergence across the Drosophila genus. The Maximum Likelihood (ML) estimate of the total tree length (using PAML software) for synonymous substitutions per synonymous site, $d_{\mathrm{s}}$, is 1.96 and 2.98 , for frq 1 and frq2, respectively. As expected from an independent evolution after gene duplication, the ML average estimate of synonymous divergence between paralogues $\left(d_{\mathrm{s}}=1.78\right)$ is higher than across orthologues (frq1, $d_{\mathrm{s}}=0.58$; frq2, $\left.d_{\mathrm{s}}=1.07\right)$, with little evidence for concerted evolution (based on a gene conversion analysis; data not shown). Surprisingly, despite the extensive divergence at synonymous sites, no nonsynonymous substitution has been detected across orthologous copies of neither frq 1 nor frq2. In fact, non-synonymous divergence is exclusively restricted to between duplicates $\left(d_{\mathrm{N}}=0.036\right)$, resulting in the 10 fixed amino acid differences between Frq1 and Frq2 proteins previously detected in [9]. Furthermore, estimates of silent variation suggest that $f r q 1$ and $f r q 2$ have evolved at different evolutionary rate across the Drosophila genus since results of the two-cluster test confirm that frq 2 accumulates synonymous substitutions faster than its paralogue (two-cluster test in LINTRE, $P$-value $=0.007)$. Using BEAST, we have estimated the age of duplication in 104 My (95\% posterior density: 78$135 \mathrm{My}$; Figure 2), which implies that the two copies originated at some point before the split of the Drosophila and Sophophora subgenera ( 60 Mya). 


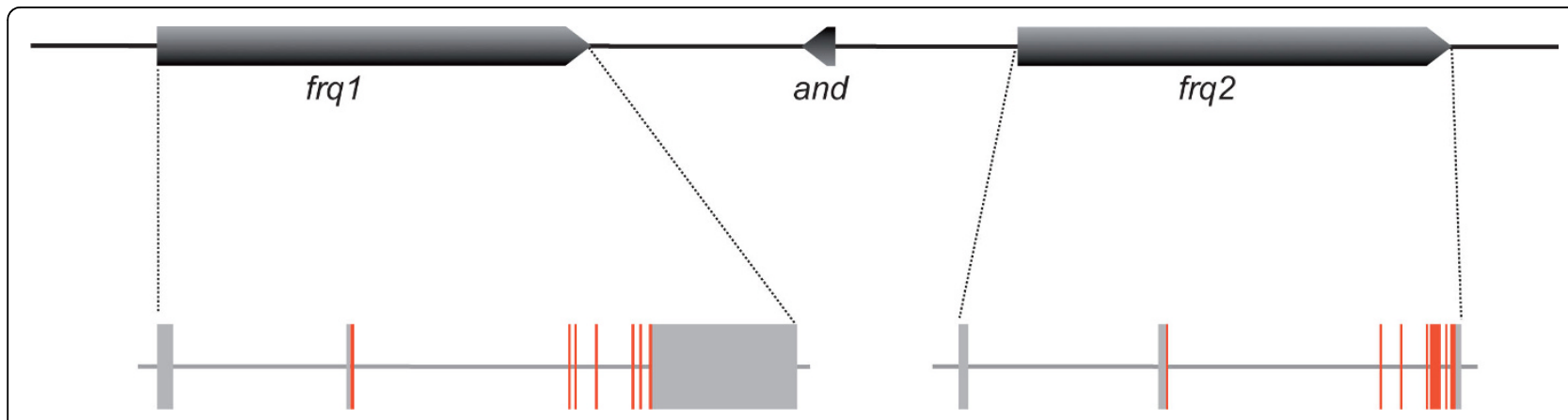

Figure 1 Genomic structure of frq1 and frq2 genes in Drosophila melanogaster. Schematic representation of the genomic region encompassing fra1 and fra 2 in the Drosophila melanogaser X chromosome. Red and grey boxes indicate coding and non-conding exons, respectively. Arrow head indicates direction of transcription with respect to the centromere which is at the right. The small gene Andorra (and) is located between the two fra genes.

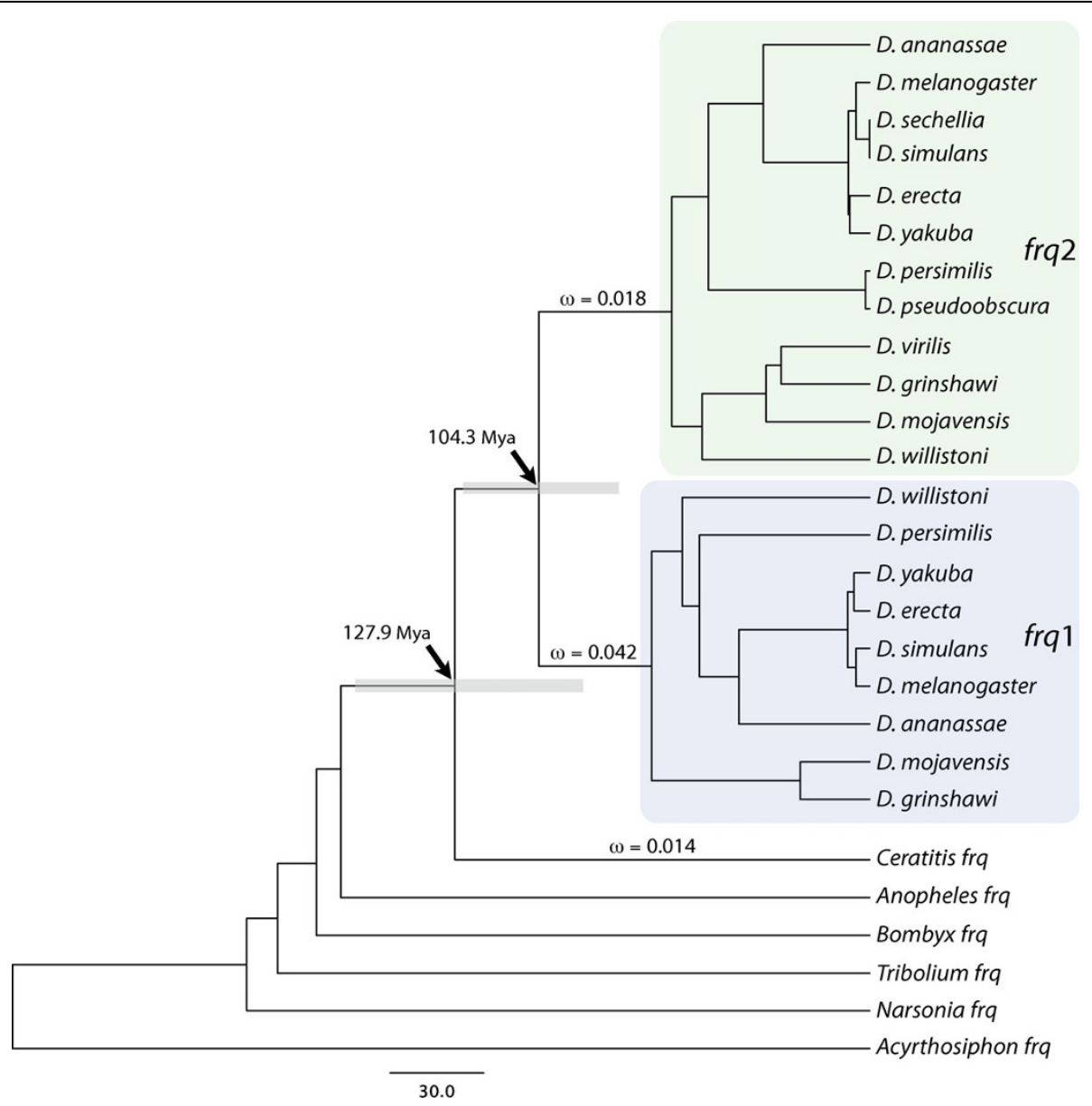

Figure 2 Phylogenetic tree of insect frq homologuous genes. Bayesian phylogeny of insect homologues based on substitutions at synonymous positions. Arrows indicate the age of the Frq1-Frq2 duplication and of the common ancestor of Drosophila and Ceratitis Frequenins. The $95 \%$ high posterior density intervals of node ages are depicted in grey. Coloured shaded boxes denote the fra 1 and fra 2 subtrees. The $\omega$ values estimated in the ML analysis are also shown. Scale is in My. 


\section{Accelerated evolution of Frq1 in early stages after gene duplication}

In contrast to Drosophila, the other surveyed insect species hold only one frq gene (Figure 2). All these single insect homologues are co-orthologues of Drosophila frq1 and frq2 (i.e., they are orthologues of both Drosophila copies), likely indicating that the duplication postdated the split between Drosophila and Ceratitis relatives. The putative sequence of the ancestral Frequenin, reconstructed by a ML approach (with PAML software) using the JTT distance [13] (which correspond to the best-fit amino acid substitution model in the model selection analysis), was used to polarize the 10 replacements fixed between duplicates; interestingly, nine of them occurred in the Frq1 lineage (only the substitution in the position 58 seems to have occurred in Frq2; amino acid positions are numbered relative to the sequence of Frq1, a convention used throughout this report). In fact, amino-acid substitution rate is significantly different between paralogues after duplication (using Ceratitis capitata Frequenin as reference and the JTT distance; Relative Rate Test module in Hyphy package, $P$-value $=0.05$ ), which is consistent with an acceleration of amino-acid substitution rate in Frq1. Replacements are broadly distributed along the primary structure of Frq1 with three of them included within EF-hand domains. Figure 3 shows the location of the residues with nonsynonymous substitutions in the modelled 3D structure. There is no apparent spatial coupling among residues, except for the two pairs that are contiguous (161 and 162) or very close (91 and 94) in the primary structure. We have also investigated the relationship between amino acid substitutions and residue accessibility. We have found that five residues (positions $5,58,94,102$ and 138) should be exposed in the Frequenin ancestral protein whereas the rest $(79,91,161$ and 162) were probably less accessible. Thus, in contrast to the observations for other proteins [14], solvent exposure does not seem to be a major structural determinant of Drosophila Frequenins residue evolution.

To determine whether functional constraints changed after gene duplication we have performed a ML analysis using the codon substitution models implemented in PAML software (Figure 4). The evolutionary scenario allowing different nonsynonymous to synonymous substitution rates ratio, $\omega$, in the internal branches leading to the frq 1 and frq 2 lineages (Model 3 ) clearly shows the best-fit to the data (Model 2 versus Model 1, $P$-value = $1.92 \times 10^{-7}$; Model 3 versus Model 2, $P$-value $=0.0002$ ). This result implies that selective constraints acting on the two copies changed both immediately after the duplication event and some time before the split of the Drosophila genus species. ML estimates of the $\omega$ parameter in these lineages (Figure 2) advocate for a relaxation of functional constraints acting on frq1 gene just after gene duplication or, alternatively, for the action of positive selection driving some of the replacements found in the protein encoded by this gene.

\section{Divergence of non-coding frq 1 and frq 2 sequences}

The evolutionary fate of duplicated copies can be also determined by changes in non-coding regions (i.e. regulatory divergence). We have analyzed nucleotide divergence at non-coding sequences of frq 1 and frq 2 in six species of the Sophophora genus. The Dot Plot of the complete genomic region containing both genes (Additional file 1) clearly shows coding exons as the only regions with significant sequence similarity between both genes, i.e. intron and flanking non-coding sequences do not retain signals of the duplication event. In contrast, the phylogenetic shadowing analysis using PhastCons reveals a number of significantly conserved non-coding sequences (CNS) across species, which might indicate the presence of functional regulatory elements (Additional file 2). Interestingly, some of these CNS are located in the two large introns of these genes (see Figure 1), which might have been maintained across Drosophila genus because of their functional role. Among these CNS, we have identified a number of topologically highly accessible transcription factor binding (TF) motives that could be involved in the regulation of frq1 and frq 2 in these species (Additional file 2). Interestingly, the motives for two factors, DL-2 (dorsal2) and Brc (Broad-complex) are only present in frq1 whereas binding sites for Eip74EF (Ecdysone induced protein 74EF) appear exclusively in frq2. Although a few species have putative binding sites for Brc and Eip74EF factors in one duplicate, in all cases only one of the sites maintains its position across the subgenus. Apart from these differentially distributed TF binding sites, the regulatory regions of frq genes have also highly accessible conserved sites for Ubx (Ultrabitorax), HB (Hunchback), Sna (Snail) and HSP-1 (Heat Shock Protein 1). In addition, we have found a number of accessible CNS with no apparent TF binding site present in surveyed databases, which might represent regulatory regions for other factors not yet identified.

\section{Amino acid sequence evolution in the NCS family}

In order to better understand the evolutionary history of Frequenin in Drosophila, we have also analyzed protein sequence evolution of this subfamily in a large set of bilaterian species. The phylogenetic analysis (Multiple Sequence Alignment (MSA) with the 38 Frequenins with complete sequence) shows that Frq/NCS-1 is a strongly conserved protein - the mean expected number of amino acid substitutions per amino acid site is 1.037. The most variable positions are outside the EF-hand 


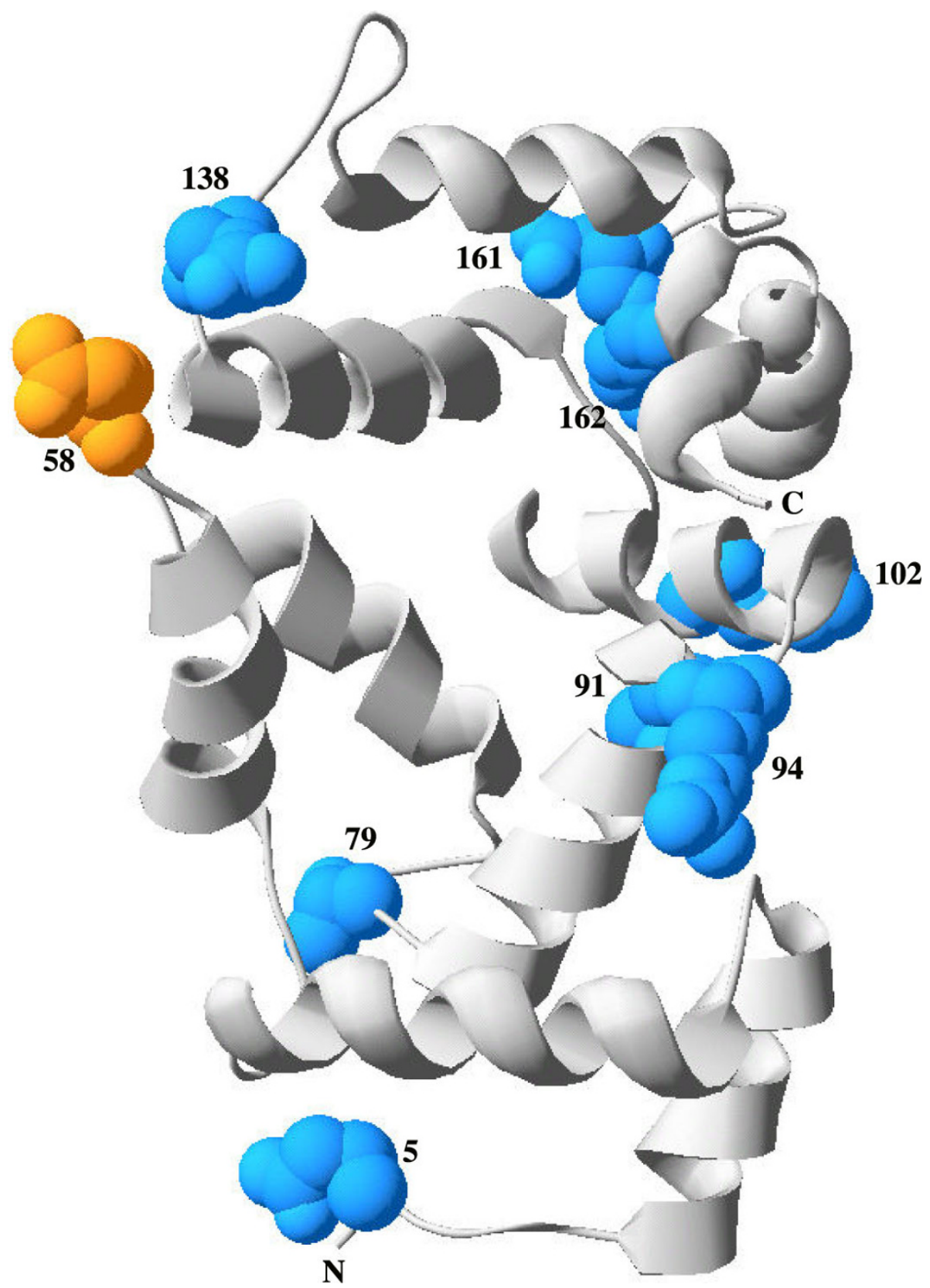

Figure 3 Putative structure of the ancestral Frequenin. Predicted 3D structure of the ancestral Frequenin. The model is based on amino acid sequence reconstructed from the comparison of known Frequenins in insects (sequences in figure 2). Amino acid replacements found in $D$. melanogaster Frq1 and Frq2 are highlighted in blue and orange, respectively. Numbers indicate the position in the Frq1. C and N termini are indicated.

domains, which are well conserved. The evolutionary rate of positions with changes between Drosophila Frequenins is variable, some of them being among the most conserved positions (Additional file 3). Results of the exhaustive search for homologous copies in databases (we found homologues of Frequeninin more than 50 bilaterian species, with representatives in major taxonomic groups) confirm that duplications are rare in the evolution of this subfamily; in fact, no fixed duplication has been found other than the already known in Danio rerio [10]. The two zebrafish copies ( $n c s 1 a$ and $n c s 1 b$ ) are noticeably younger than $f r q 1$ and $f r q 2$, i.e. the closest species in our data set with only one Frequenin is Pimephales pomelas, which diverged from Danio rerio 20-50 Mya [15,16]; NCS1a and NCS1b differ in 7 amino acid positions, but unlike in Drosophila, changes have been 


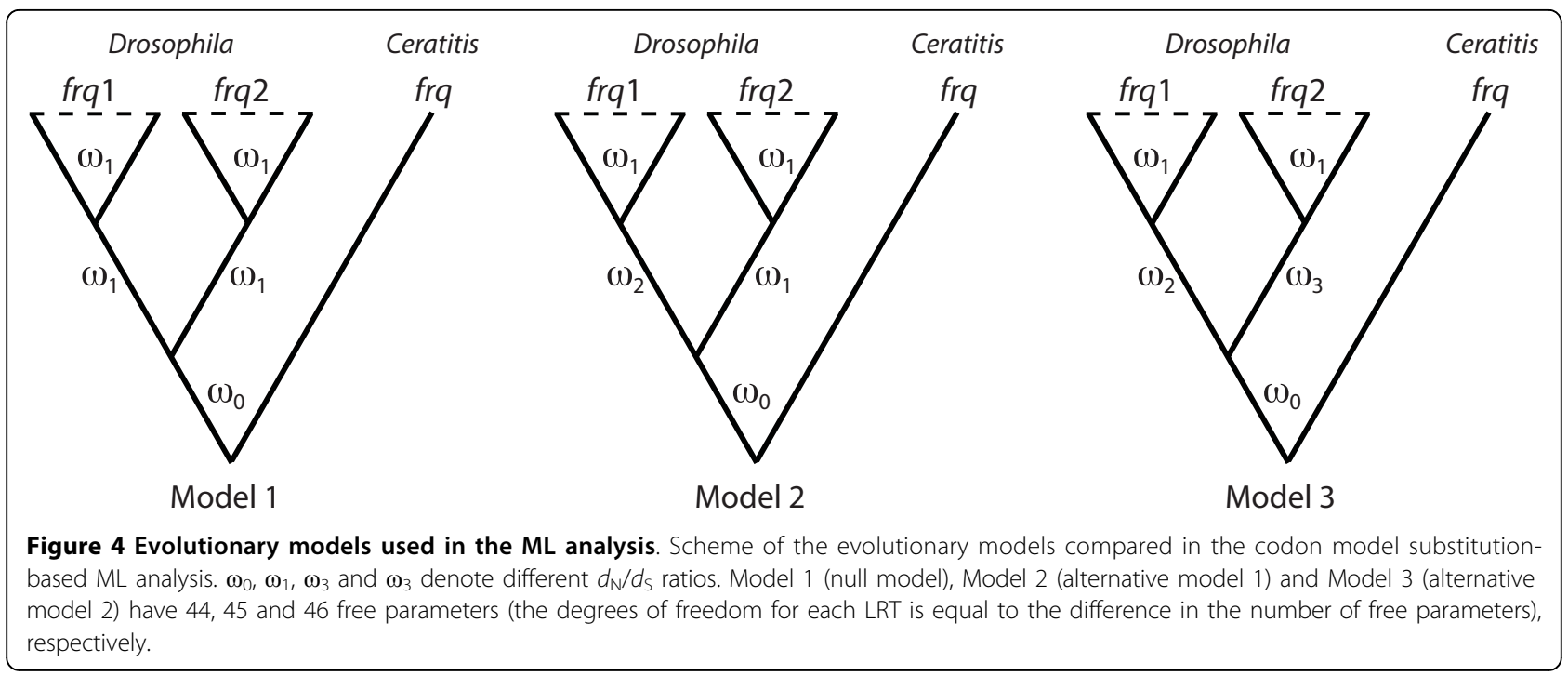

homogeneously distributed between paralogues after gene duplication (using the sequence of $P$. pomelas as reference, 3 and 3 changes in NCS1a and NCS1b, respectively, and one ambiguous replacement).

To evaluate the possibility that amino acid substitutions between Frq1 and Frq2 were correlated, we have searched for the presence of coevolving positions across the Frq/NCS-1 subfamily MSA. The analysis based on correlation coefficients [17] does not detect any significant intra-molecular co-evolution across bilaterian Frequenins. In contrast, the CTMP model approach [18], allows detecting 40 pairs of amino acid sites with significantly more double substitutions than expected under the best-fit amino acid substitution model. The predicted positions are scattered across the sequence and only the C-terminal is free of putative co-evolving positions. No coordinating residues of the EF-hands appear among the sites with high score, with the only exception of position 79, located at the second EF hand. Some of the predicted coevolving pairs enclose positions with amino acid differences between Frq1 and Frq2 proteins (positions 58, 79, 102 and 138 in Drosophila Frq1). Nevertheless, one of these positions (position 58) corresponds to the substitution in the Frq2 lineage and no significant pair is composed by two sites with replacements in Frq1.

We have investigated whether amino acid substitutions between Frq1 and Frq2 are actually involved in functional divergence (rather than representing compensatory changes). For that, we extended the analysis to the other NCS subfamilies. The rate of evolution is remarkably different both among subfamilies and between the members of the same NCS class (we analyzed the 14 subfamilies that compose the $5 \mathrm{NCS}$ classes; Table 1 in [6]). NCS-1 and VILIP1 are clearly the most conserved subfamilies (the average root-to-tip length of the vertebrate tree for amino acid substitutions per site is 0.017 and 0.025 , respectively) whereas GCAP subfamilies evolve faster than the rest (ranging from 0.297 to 0.739 ). The results of the functional divergence analysis in Diverge software reveal that positions 58, 79, 91, 102, 138, 161 and 162, have been involved (they are among the $5 \%$ highest values in the posterior probability profiles) in functional constraints changes and specific functional divergence between NCS subfamilies (Table 1 ). These positions show site-specific shifts of evolutionary rate (type-I functional divergence) and/or of amino acid property (type-II functional divergence). Interestingly, although the specific amino acids present in positions 79, 102 and 162 of Frq1 and Frq 2 are not the same than those responsible for type II functional divergence between NCS proteins, they also denote radical changes in Frq1 (i.e. in terms of charge and hydrophobicity). This suggests that they might have been involved in cluster specific functional divergence between Frq1 and Frq2 [19]. The same occurs with position 58, which imply a radical change in Frq2. The putative contribution of the positions 5 and 186 to functional divergence between subfamilies was unachievable because of the high levels of divergence in the $\mathrm{C}$ - and $\mathrm{N}$-terminal parts of the NCS proteins. The rapid evolution of these regions prevented a confident identification of positional homologies for the two sites.

Among the amino acid positions replaced between Frq1 and Frq2, positions 58, 79, 102, 161 and 162 are the more firm candidates to participate in the functional diversification of the NCS family (Table 1). These five positions are involved in many independent rate shift changes across the evolution of NCS members, although the particular amino acid residues and the type of 
Table 1 Functional divergence between NCS subfamilies

\begin{tabular}{|c|c|c|c|c|}
\hline Subfamily (Class) & Subfamily (Class) & Type I $\theta$ (SE) & Type II $\theta$ (SE) & Positions $^{\mathrm{a}}$ \\
\hline \multirow[t]{8}{*}{ Frq/NCS-1 (A) } & Recoverin (C) & $0.246(0.089)$ & $0.342(0.072)$ & $79,102,162$ \\
\hline & Hippocalcin (B) & $0.858(0.230)$ & $0.273(0.046)$ & $79,102,162$ \\
\hline & KChIP1 (E) & $0.899(0.233)$ & $0.415(0.063)$ & $58,79,102,138$ \\
\hline & KChIP2 (E) & NS & $0.348(0.078)$ & 58 \\
\hline & KChIP3 (E) & $0.594(0.268)$ & NS & 138,162 \\
\hline & KChIP4 (E) & $0.561(0.232)$ & $0.447(0.057)$ & $58,138,162$ \\
\hline & VILIP2 (B) & NS & $0.298(0.052)$ & 102 \\
\hline & VILIP3 (B) & $0.829(0.285)$ & - & 79,102 \\
\hline \multirow[t]{8}{*}{ Recoverin (C) } & VILIP2 (B) & NS & $0.317(0.076)$ & 102 \\
\hline & VILIP3 (B) & $0.563(0.187)$ & NS & 102 \\
\hline & GCAP1 (D) & $0.665(0.069)$ & $0.480(0.088)$ & 79,161 \\
\hline & GCAP2 (D) & $0.765(0.076)$ & $0.501(0.077)$ & 161,161 \\
\hline & KChIP1 (E) & $0.778(0.090)$ & NS & 91 \\
\hline & KChIP2 (E) & $0.867(0.095)$ & NS & 79 \\
\hline & KChIP3 (E) & $0.665(0.070)$ & $0.547(0.074)$ & 79,161 \\
\hline & KChIP4 (E) & $0.731(0.100)$ & $0.500(0.070)$ & $79,161,162$ \\
\hline \multirow[t]{3}{*}{ Hippocalcin (B) } & KChIP1 (E) & NS & $0.455(0.060)$ & 58 \\
\hline & KChIP2 (E) & NS & $0.430(0.073)$ & 58 \\
\hline & KChIP4 (E) & NS & $0.496(0.055)$ & 58,162 \\
\hline \multirow[t]{4}{*}{ VILIP2 (B) } & KChIP1 (E) & NS & $0.483(0.060)$ & 161 \\
\hline & KChIP2 (E) & NS & $0.517(0.066)$ & 161 \\
\hline & KChIP3 (E) & NS & $0.416(0.076)$ & 161 \\
\hline & KChIP4 (E) & NS & $0.546(0.055)$ & 161 \\
\hline GCAP3 (D) & GCAP2 (D) & NS & $0.498(0.127)$ & 79 \\
\hline
\end{tabular}

a Sites with changes between Frq1 and Frq2 that show significant shift in their evolutionary rate between NCS subfamilies (type I and type II sites are in regular and italic font, respectively). Positions are numbered relative to the $D$. melanogaster Frq 1 protein. $\theta, \mathrm{ML}$ estimate of the coefficient of functional divergence. SE, standard error. NS, non-significant.

divergence depend on the comparison. Position 58 contributed to the functional divergence between members of the class $E$ and representatives of the classes $A$ and B. Positions 79 and 102 were involved in several rate shift changes associated with functional divergence between all NCS subfamilies and between families belonging to the classes $\mathrm{A}, \mathrm{B}$ and $\mathrm{C}$, respectively. Position 161 is a functional diverged site between KChIPs and other subfamilies, whereas the contiguous position, 162 , is among the sites with high posterior probability in different comparisons between all NCS classes. Interestingly, these five positions, which involve chemically radical changes between Frq1 and Frq2, also participated (apart from in type I) in type II functional divergence between NCS subfamilies. Finally, positions 91 and 138 (with chemically conservative changes in Frq1) are candidates only for type I functional divergence (between KChIPs and Frequenin and Recoverin, respectively).

\section{Discussion}

The NCS family includes members with very different evolutionary rates, which provide insights into their diversification in both structure and function. Among these calcium sensors, Frq/NCS-1 appears to be one of the most conserved subfamilies, not only at the amino acid sequence level but also because of their very low propensity to retain gene duplications - no other fixed duplication has been detected here except in $D$. rerio. For that reason, the discovery of a fixed and long-term stable (estimated as > 100 Mya) duplication in Drosophila, as well as the rapid and heterogeneous accumulation of amino acid changes between duplicates, constitutes a very unusual feature for this family. Understanding the basis of this finding may contribute to the better knowledge of molecular evolution of NCS proteins as well as of important fields as the origin and fate of duplicated genes and of functional innovation.

Gene duplications arise initially in a single individual and can become fixed in the population by natural selection or by random genetic drift. Initially, duplications generate functional redundancy, a situation that is generally non-advantageous; hence, even if duplicates have been fixed by chance, the accumulation of mutations will result in the disruption of structure and function of one of the duplicates, which becomes a pseudogene (i.e. a non-functional gene). Results of nucleotide and amino acid variation analyses performed here clearly demonstrate that amino acid changes 
observed in Drosophila Frequenins are not the result of this pseudogenization process. The coding sequence of frq1 shows strong functional constraints across the Drosophila genus as well as within a $D$. melanogaster population (unpublished results) and none of the nine amino acid substitutions affecting the encoded protein was disruptive, pointing to purifying selection as the main force acting on this gene. In addition, analysis of loss-of-function and over-expression phenotypes also point to the role of both Frq1 and Frq 2 on synaptic transmission and nerve terminal growth $[9,11]$.

Several theoretical models have been proposed to explain the maintenance of duplicated genes, which consider different mechanisms of preservation and subsequent optimization [20-25]. The main differences among models rely in which part of the gene is involved (coding or noncoding sequences) and in the relative role of natural selection and genetic drift in determining the outcome of duplication. Dealing with protein sequence evolution, two duplicates can be preserved without the action of positive selection just by the selectively neutral division of different subsets of the original functions between daughter copies (product subfunctionalization). Nevertheless, this situation is not compatible with present data because of: i) the distribution of mutations after gene duplication is significantly different from the one expected under neutrality, and ii) the strongly selective constrains acting on amino acid positions affected by these mutations across the Drosophila genus. We would expect that positions with degenerative mutations removing ancestral functions evolved in a completely neutral way.

The amino acid substitution pattern observed in Frq1 might result from the fixation of nearly neutral mutations (and so governed largely by genetic drift) in an initial period of relaxation, just after gene duplication, followed by a subsequent increase of functional constraints in Drosophila. Environmental conditions responsible for these constraint changes would affect frq 1 and frq 2 in a different way as suggested by the highly biased distribution of amino acid substitutions detected between these paralogues.

Accordingly, some functional or regulatory diversification from the native state between Drosophila Frequenins would be needed. In this context, some models of structural evolution such as compensatory mutations [26] or conformational epistasis [27] might generate the observed evolutionary pattern. In the first, some variants are fixed by positive selection to compensate deleterious mutations in other epistatically interacting positions. Under this model, some of the amino acid substitutions in Frq1 ought to have been selected to maintain protein structure or function rather than be adaptive [28]. Under the conformational epistasis hypothesis, most of the Frq1 mutations should have been slightly deleterious or permissive substitutions (i.e. small-effect) that stabilized specific structural elements in this protein allowing further positively selected mutations-which in the absence of previous small-effect mutations should destabilize the protein. These mutations could have conferred a new function and then increased selective constraints in Drosophila. Although we have found evidences of molecular co-evolution across the Frq/NCS-1 subfamily, none of the predicted coevolving amino acids involves two positions with replacements in Frq1. This finding should rule out structural evolution as the main explanation for the rapid accumulation of amino acid substitutions between duplicates. Nevertheless, the probability of observing more double substitutions than expected by chance largely depends on the presence of relatively strong epistatic interactions between mutations. If both Frequenins have very few potentially permissive substitutions, the probability of observing repeated pathways across the subfamily should be very low. Under this situation, we will have very little power to detect signals of co-evolution between amino acid sites across the alignment. An in-depth experimental study would be needed to analyze the putative contribution of these structural evolution models in generating the pattern observed in Drosophila Frequenins.

The recurrent fixation of advantageous mutations might also account for the excess of amino acid substitutions in Frq1. ML estimate of the $d_{\mathrm{N}} / d_{\mathrm{S}}$ ratio in the internal branch leading to Drosophila Frq1 sequences is actually higher than in the rest of the branches of the Drosophila Frq phylogeny. Although the estimate is considerably lower than 1, as well as lower than genomic averages reported for the Drosophila genus [29], this result does not exclude the possibility that positive selection acted in the fixation of certain Frq1 changes. It has been largely demonstrated that positive selection commonly acts on few amino acid positions in a protein and, therefore, present estimates based on the complete coding sequence could be too conservatives. Frequenin is a highly conserved protein, with very low $\omega$ estimates across bilateria (the pair-wise $\omega$ ratios calculated between vertebrate NCS1 sequences range from 0 to $0.0065 ; d_{\mathrm{N}} / d_{\mathrm{S}}$ data from Ensembl Genome Browser) and, therefore, even a significant increase in the number of amino acid changes could not be greatly reflected in the average $\omega$ value calculated from the entire protein. In fact, when we applied to the data a much more powerful branch-site approach [30], results were marginally significant (results not shown). Even so, we have to interpret this result with caution because it has been reported that this method often generates false positives under certain conditions [31,32].

All simple models for the preservation of frq 1 and frq 2 in Drosophila considered above that are compatible with the existing data require the action of positive selection. 
Thus, the key question that remains unsolved is if natural selection promoted a functional change between Frq1 and Frq2 in Drosophila. We investigated whether positions differing between paralogues are involved in functional diversification between other members of the NCS family. The fact that many of these positions are candidates to participate in significant amino acid substitution rate shifts between NCS subfamilies suggests that Frq1 and Frq2 might have diverged (at least to some extent) in their functions. It is difficult to determine, however, the specific functional features that could have diverged between these two proteins. Most of the positions with changes between Drosophila Frequenins are among the most probable functionally diverged residues in many subfamily comparisons. The putative specialized roles in neural function of Drosophila Frequenins might result from differences in the affinity to $\mathrm{Ca}^{2+}$, sub-cellular location or targeted proteins [6]. It has been demonstrated that sites of the C-terminal part of the human NCS-1 interact with target proteins [33]. Also, we had shown that the last 33 amino acids of the Drosophila Frq1 and Frq2 act as dominant negative peptide, with effects in synaptic transmission and terminal morphology [9]. Thus, the two candidate C-terminal positions, 161 and 162 (and perhaps the position 187) might have promoted some diversification in the interaction with target proteins. On the other hand, the replacements observed in these two sites might have also produced changes in $\mathrm{Ca}^{2+}$ binding either directly, because they are located in the fourth EF-hand, or by producing structural changes affecting protein thermostability and $\mathrm{Ca}^{2+}$ affinity of the other EF-hands [34-36]. The position 79 is one of the coordinated residues of the second EF-hand and, therefore, replacements in this site might also be related with $\mathrm{Ca}^{2+}$ affinity differences between paralogues. The amino acid fixed in Frq1 in this position is hydrophilic and highly exposed, in contrast to the hydrophobic and buried ancestor. This feature might indicate a possible structural change produced by the replacement in this position. Binding sites for target proteins have been also mapped in the N-terminal part of the human Frq/NCS1protein [37]. Consequently, substitution in positions 58 (and perhaps in 79 and 102 in Frq1) might have altered the interaction properties of Frq2 with some of their partners. The other good candidate to participate in functional divergence between NCS subfamilies, the position 102, is located in the loop connecting the second and third EF-hands. The homologous region in GUCA2 determines the concentration of $\mathrm{Ca}^{2+}$ that activates the target of this protein [38]. Then, the replacement in this position might also affect the $\mathrm{Ca}^{2+}$ binding properties of Frq1, contributing to the functional divergence of duplicates in Drosophila.
Finally, in addition to the retention and diversification of protein coding regions, regulatory divergence is also prevalent in the evolution of duplicated genes [39]. In fact, it has been proposed that subfunctionalization of regulatory regions can increase the mutational space accessible to duplicates, removing selective constraints and allocating neofunctionalization [40]. The mRNAs of frq 1 and $f r q 2$ are expressed in D. melanogaster with a similar spatio-temporal pattern, but with important quantitative differences [9]. These quantitative differences could be related with the significantly different silent evolutionary rate found between these two proteins since it has been shown that gene expression levels are negatively correlated with evolutionary rates [41]. Here we found that Brc and Eip74EF factors might be involved in the regulatory divergence of these two duplicates. Suitable Bcr binding sites are only present in regulatory regions of $f r q 1$. In fact, $f r q 1$ and $b r$ (the locus encoding Brc proteins) [42] mRNAs have a very similar expression pattern in late stages of embryo development (both appear in brain and ventral nervous system at embryo stages 13-16; http://www.fruitfly.org/cgi-bin/ex/ insitu.pl), coincident with the peak of $f r q 1$ expression. Eip74EF, in contrast, appear only in early metamorphosis, after the major pulse of Ecdysone in the third larval instar, being consistent with the higher expression of frq 2 mRNA in adult flies. Hence, differences in the response to these two Ecdysone-induced early genes might be responsible, at least in part, for the differences in gene expression levels between duplicates. This regulatory diversification might render the action of positive selection suitable, resulting in further functional diversification at the protein level. The current in silico analysis sets the frame of future experimental studies on the regulation of $f r q 1$ and $f r q 2$ expression, as well as on the functional mechanisms of the corresponding proteins.

\section{Conclusions}

The increasing availability of sequenced genomes from polymorphic to highly divergent taxa has allowed the application of phylogenetic and molecular variation analyses across different time scales. This approach has proven useful to disentangle evolutionary processes that were not completely resolved in previous comparative genomic or population genetics studies. Using this approach we found that positive selection, acting on protein sequence evolution, jointly with the diversification of non-coding regulatory sequences, might be the main forces responsible for the origin and preservation of the unusual Frequenin duplication in Drosophila. The results of this study of variation at the molecular level of Frequenin sequences will inspire future experiments on the mechanisms for protein function and gene regulation. 


\section{Methods}

\section{Sequence searches}

We used TBLASTN tool [43] against public databases to search homologues of the Drosophila Frq1 and Frq2 proteins. The databases surveyed were: i) the twelve complete Drosophila genomes available in FlyBase [44] ii) the genomes of invertebrate vectors of human pathogens in VectorBase [45], iii) the dbESTs database in GeneBank [46] and iv) eukaryotic genomes available in ENSEMBL project [47]. In addition, we retrieved all proteins annotated as members of the NCS family from ENSEMBL database (family accession number: ENSFM00500000269655). Orthologous relationships were assigned using the BLAST reciprocal best-hit approach together with gene tree and species tree reconciliation and synteny conservation. Gene structure features of Drosophila orthologues were revisited and adjusted using the information of FlyBase annotated genes (http://rana.lbl.gov/drosophila/; Drosophila 12 Genomes Consortium) as a guide.

\section{Nucleotide sequence analyses}

The DNA sequences corresponding to the coding regions of $f r q 1$ and $f r q 2$ genes were multiple aligned using the Mafft software [48], and manually edited with MacClade version 3 program [49]. The resulting multiple sequence alignment (MSA) was used to estimate nucleotide sequence variation in DnaSP version 5.0 [50], and MEGA version 4 [51] software. To determine if paralogous genes evolve at different substitution rates we conducted the two-cluster test implemented in the LINTRE package [52].

BEAST version 1.4.8 [53] was used to place the duplication event that produces frq1 and frq 2 copies on the insect phylogeny and to obtain a posterior density of the duplication age. The tree was built using the 21 complete Drosophila coding regions available plus the sequence of 6 other insects. We set constraints on divergence times of i) the Drosophila and Sophophora subgenera, ii) the melanogaster and obscura groups and iii) the divergence of the species belonging to the melanogaster subgroup. These ages were set in uniform distributions with ranges previously published [54]. We used divergence at synonymous sites with a relaxed, uncorrelated lognormal clock and the HKY85 nucleotide substitution model [55] with gamma rate heterogeneity. The length of the Markov chain was 5000000, with a sample frequency every 200. The first 2500 trees were discarded in the burn-in step.

Selective pressures acting on coding regions after gene duplication and the estimates of synonymous and nonsynonymous rates, including total tree length for synonymous substitutions, were estimated using the ML approach implemented in PAML version 4 [56]. Since homologous sequences identified in mosquito and Drosophila are highly divergent (they might yield unreliable estimates of the relevant parameters) we used only the 21 Drosophila coding regions and the sequence of the fly Ceratitis capitata for the analysis. The ratio of nonsynonymous $\left(d_{\mathrm{N}}\right)$ to synonymous substitution rates $\left(d_{\mathrm{s}}\right)$, $\omega=d_{\mathrm{N}} / d_{\mathrm{S}}$, was used as the measure of protein selective constraints [57]. Competing models (Figure 4) were compared in a Likelihood Ratio Test (LRT), assuming that twice the log likelihood difference between the two models $(2 \Delta \ell)$ follows a $\chi^{2}$ distribution with a number of degrees of freedom equal to the difference in the number of free parameters [58]. To prevent incorrect parameter estimates caused by local optima, the program was run multiple times for the same model, specifying different initial values.

In order to identify the regions of DNA sequence similarity produced by the duplication event, a Dot Plot of the complete genomic region $(\sim 66 \mathrm{~kb})$ that includes frq1 and frq2 (and andorra) genes in D. melanogaster was obtained in the Zpicture tool web [59]. Moreover, a MSA corresponding to this genomic region in 6 Drosophila genomes (D. melanogaster, D. simulans, D. erecta, D. yakuba, D. ananassae, and D. pseudoobscura) was obtained from the pre-computed alignments of Comparative Assembly Freeze 1 (CAF1) http://rana.lbl.gov/ drosophila/caf1.html available in VISTA server [60]. This MSA was used to perform a phylogenetic shadowing [61] search for conserved (orthologous) non-coding regions using the hidden Markov model (phylo-HMM) approach implemented in PhastCons [62]. We used the UCSC genome browser database [63] aligment of all CDSs in the genomic fragment corresponding to the positions chrX:2000000-3000000 for modelling sequence conservation. The same MSA was also analysed with the Chai algorithm to obtain a profile of protein binding affinity based on local DNA topology [64]. Finally, regions selected as candidates to include functional regulatory elements were scanned for the presence of insect transcription factor binding site profiles contained in TRANSFAC [65] and JASPAR [66] collections.

\section{Amino acid sequence analyses}

For protein sequence analyses, we built two different MSA. The first (Additional file 4) included all bilaterian members of the Frequenin subfamily retrieved from databases and was used to analyze amino acid co-evolution by means of two approaches. In one of them, the correlated variance of the evolutionary rates between amino acid sites was calculated in CAPS software [67]. In the other, a ML approach [18] based in a continuous time Markov substitution process (CTMP) was applied 
to the data. This method compares the goodness of fit of a null model, in which each pair of sites evolves independently, and an alternative coevolving model obtained by reweighing the independent substitution rate matrix to favour double over single changes. For this analysis, we used a $\varepsilon$ value of 0.75 and only the positions with a log-odds score threshold $>15$ were considered. The second protein MSA, with only vertebrate sequences but with representatives of the entire NCS family, was used to identify the amino acid positions with significant shifts in substitution rates, likely responsible for changes in functional constrains and functional diversification between NCS subfamilies. We analyzed only vertebrate sequences to avoid large unbalanced trees (i.e., only vertebrates have representatives of all NCS subfamilies), which can produce biased results [19]. We discarded sequences representing species or lineage specific expansions, which likely reflect particular histories, partial sequences and sequences that produce poor aligned fragments (Additional file 5). Type I and type II functional divergence coefficients $[68,19]$ as well as the posterior probability (PP) profiles to contribute to functional divergence of all amino acid sites were calculated in Diverge 2.0 software [69]. ML trees for these analyses were built in PhyML [70] using the best-fit amino acid substitution model selected in MODELTEST 3.7 [71]. Sites with the 5\% highest $P P$ values were considered as firm candidates to be functionally diverged sites. Functional divergence between KChIP and GCAP subfamilies was not analyzed because of the high level of divergence and the poor quality of the alignment.

Neurocalcin- $\delta$ and VILIP1 subfamilies were not used for this analysis because of their lack of amino acid rate heterogeneity. The probability of observing a given distribution of amino acid changes between paralogues after gene duplication was calculated with the Relative Rate Test module implemented in Hyphy package [72]. This test was performed using the previously selected amino acid substitution model.

The putative 3D structure of the ancestral Frequenin (inferred by ML ancestral reconstruction in PAML 4) was obtained with Swiss-Model server [73]. The 3D structure of human Frequenin (PDB ID: 1G8I) [74,75] was used as template for this analysis. The model was visualized in Swiss-PdbViewer program version 4 [76]. This program was also used to highlight the relevant amino acid replacements identified in the evolutionary analyses.

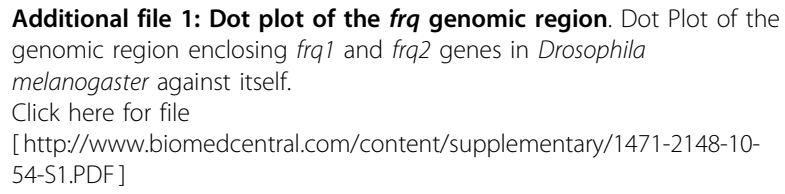

Additional file 2: Conservation, DNA topology and transcription factor binding sites predictions. Conservation, DNA topology and transcription factor (TF) binding sites profiles of the different fral and fra 2 non-coding regions analyzed. Red and blue lines indicate sequence conservation across species and DNA accessibility, respectively. The $\mathrm{Y}$-axis indicates either the posterior probability of each individual site to be conserved relative to the genomic fragment used as reference in PhastCons [62] or the normalized score (the score of each site divided by the maximum of the investigated region) obtained in Chai [64]. Vertical coloured bars indicate the conserved elements predicted by the Viterbi algorithm in PhastCons (the probability of conservation increases from blue to red). Horizontal bars indicate the positions predicted to contain insect TF binding site profiles present in TRANSFAC [65] and JASPAR [66] collections. Click here for file

[http://www.biomedcentral.com/content/supplementary/1471-2148-1054-S2.PDF ]

Additional file 3: Amino acid evolutionary rates in NCS-1 subfamily. Distribution of amino acid evolutionary rates across NCS-1 protein. Asterisks show the amino acid positions that differ between Frq1 and Frq2. The evolutionary rate in these positions is coloured in grey. Click here for file

[http://www.biomedcentral.com/content/supplementary/1471-2148-1054-S3.PDF ]

Additional file 4: Frq/NCS-1 subfamily multiple sequence alignment. Multiple sequence alignment with all bilaterian NCS-1 sequences used in the amino acid sequence evolution analysis of this subfamily. Data is in NBRF format.

Click here for file

[http://www.biomedcentral.com/content/supplementary/1471-2148-1054-S4.DOC]

Additional file 5: Data set of vertebrate NCS family sequences. Data set of vertebrate NCS sequences used in the functional divergence analysis. Species names are in the same four-letter format as in pre-computed alignments downloaded from ENSEMBL (except for Frequenin subfamily sequences, which are named as in Additional file 4. Data is in NBRF format. Click here for file

[http://www.biomedcentral.com/content/supplementary/1471-2148-1054-S5.DOC ]

\section{Acknowledgements}

We thank J. Rozas for helpful comments and suggestions on the manuscript and P. Librado for his assistance with the evolutionary analysis of noncoding sequences. We also thank S. Parker for sharing the program Chai. AS is deeply grateful to the members of the A. Ferrús laboratory for sharing their expertise about Drosophila genetics and molecular biology. Research was funded by grants BFU 2006-10180 and the European Research Network of Excellence MYORES ref.: CE-511978.

\section{Author details}

'Instituto Cajal, CSIC, Ave. Dr. Arce 37, Madrid 28002, Spain. ${ }^{2}$ Institute of Evolutionary Biology, IBE (CSIC-UPF), Passeig Marítim de la Barceloneta, 3749, 28003 Barcelona, Spain. ${ }^{3}$ Centro de Biología Molecular, UAM/CSIC, c/ Nicolas Cabrera 1 Madrid 28049, Spain.

\section{Authors' contributions}

AS-G designed the study, carried out the analyses and wrote the first version of the manuscript. JR-P provided complementary information and modified the text. AF supervised the study and edited the final text. All authors read and approved the final manuscript.

Received: 24 August 2009

Accepted: 19 February 2010 Published: 19 February 2010

\section{References}

1. Berridge MJ: Neuronal calcium signaling. Neuron 1998, 21(1):13-26.

2. Augustine GJ, Santamaria F, Tanaka K: Local calcium signaling in neurons. Neuron 2003, 40(2):331-346. 
3. Burgoyne RD, O'Callaghan DW, Hasdemir B, Haynes LP, Tepikin AV: Neuronal Ca2+-sensor proteins: multitalented regulators of neuronal function. Trends Neurosci 2004, 27(4):203-209.

4. Braunewell $\mathrm{KH}$, Gundelfinger ED: Intracellular neuronal calcium sensor proteins: a family of EF-hand calcium-binding proteins in search of a function. Cell Tissue Res 1999, 295(1):1-12.

5. Braunewell $\mathrm{KH}$ : The darker side of $\mathrm{Ca} 2+$ signaling by neuronal $\mathrm{Ca} 2$ +-sensor proteins: from Alzheimer's disease to cancer. Trends Pharmacol Sci 2005, 26(7):345-351.

6. Burgoyne RD: Neuronal calcium sensor proteins: generating diversity in neuronal Ca2+ signalling. Nature Rev Neurosci 2007, 8(3):182-193.

7. Pongs O, Lindemeier J, Zhu XR, Theil T, Engelkamp D, Krah-Jentgens I, Lambrecht HG, Koch KW, Schwemer J, Rivosecchi R, et al: Frequenin-a novel calcium-binding protein that modulates synaptic efficacy in the Drosophila nervous system. Neuron 1993, 11(1):15-28.

8. Angaut-Petit D, Toth P, Rogero O, Faille L, Tejedor FJ, Ferrus A: Enhanced neurotransmitter release is associated with reduction of neuronal branching in a Drosophila mutant overexpressing Frequenin. Eur J Neurosci 1998, 10(2):423-434.

9. Romero-Pozuelo J, Dason JS, Atwood HL, Ferrus A: Chronic and acute alterations in the functional levels of Frequenins 1 and 2 reveal their roles in synaptic transmission and axon terminal morphology. Eur $J$ Neurosci 2007, 26(9):2428-2443.

10. Blasiole B, Kabbani N, Boehmler W, Thisse B, Thisse C, Canfield V, Levenson R: Neuronal calcium sensor-1 gene ncs-1a is essential for semicircular canal formation in zebrafish inner ear. J Neurobiol 2005, 64(3):285-297.

11. Dason JS, Romero-Pozuelo J, Marin L, lyengar G, Klose MK, Ferrús A, Atwood HL: Frequenin/NCS-1 and the Ca2+-channel $\alpha_{1}$-subunit coregulate synaptic transmission and nerve-terminal growth. J Cell Sci 122:4109-4121.

12. Clark AG, Eisen MB, Smith DR, Bergman CM, Oliver B, Markow TA, Kaufman TC, Kellis M, Gelbart W, lyer VN, Pollard DA, Sackton TB, Larracuente AM, Singh ND, Abad JP, Abt DN, Adryan B, Aguade M, Akashi $H$, Anderson WW, Aquadro CF, Ardell DH, Arguello R, Artieri CG, Barbash DA, Barker D, Barsanti P, Batterham P, Batzoglou S, Begun D, et al: Evolution of genes and genomes on the Drosophila phylogeny. Nature 2007, 450(7167):203-218.

13. Jones DT, Taylor WR, Thornton JM: The rapid generation of mutation data matrices from protein sequences. Comput Appl Biosci 1992, 8:275-282.

14. Franzosa EA, Xia Y: Structural Determinants of Protein Evolution are Context-Sensitive at the Residue Level. Mol Biol Evol 2009, 26(10):2387-2395.

15. Zardoya R, Doadrio I: Molecular evidence on the evolutionary and biogeographical patterns of European cyprinids. J Mol Evol 1999, 49(2):227-237.

16. Ruber L, Kottelat M, Tan HH, Ng PK, Britz R: Evolution of miniaturization and the phylogenetic position of Paedocypris, comprising the world's smallest vertebrate. BMC Evol Biol 2007, 7:38.

17. Fares MA, Travers SA: A novel method for detecting intramolecular coevolution: adding a further dimension to selective constraints analyses. Genetics 2006, 173(1):9-23.

18. Yeang $\mathrm{CH}$, Haussler D: Detecting coevolution in and among protein domains. PLoS Comp Biol 2007, 3(11):e211

19. Gu X: A simple statistical method for estimating type-II (cluster-specific) functional divergence of protein sequences. Mol Biol Evol 2006, 23(10):1937-1945.

20. Ohno S: Evolution by gene duplication. Berlin: Springer 1970.

21. Piatigorsky J, Wistow $\mathrm{G}$ : The recruitment of crystallins: new functions precede gene duplication. Science 1991, 252(5010):1078-1079.

22. Hughes AL: The evolution of functionally novel proteins after gene duplication. P Roy Soc B-Biol Sci 1994, 256(1346):119-124.

23. Force $A$, Lynch M, Pickett FB, Amores A, Yan YL, Postlethwait $P$. Preservation of duplicate genes by complementary, degenerative mutations. Genetics 1999, 151(4):1531-1545.

24. Zhang J: Evolution by gene duplication: an update. Trends Ecol Evol 2003, 18(6):292-298.

25. Conant GC, Wolfe KH: Turning a hobby into a job: how duplicated genes find new functions. Nat Rev Genet 2008, 9(12):938-950.

26. Kondrashov AS, Sunyaev S, Kondrashov FA: Dobzhansky-Muller incompatibilities in protein evolution. P Natl Acad Sci USA 2002, 99(23):14878-14883.
27. Ortlund EA, Bridgham JT, Redinbo MR, Thornton JW: Crystal structure of an ancient protein: evolution by conformational epistasis. Science 2007, 317(5844):1544-1548.

28. DePristo MA, Weinreich DM, Hartl DL: Missense meanderings in sequence space: a biophysical view of protein evolution. Nat Rev Genet 2005, 6(9):678-687

29. Heger A, Ponting CP: Evolutionary rate analyses of orthologs and paralogs from 12 Drosophila genomes. Genome Res 2007, 17:1837-1849.

30. Zhang J, Nielsen R, Yang Z: Evaluation of an improved branch-site likelihood method for detecting positive selection at the molecular level. Mol Biol Evol 2005, 22(12):2472-2479.

31. Suzuki Y: False-positive results obtained from the branch-site test of positive selection. Genes Genet Syst 2008, 83(4):331-338.

32. Nozawa M, Suzuki Y, Nei M: Reliabilities of identifying positive selection by the branch-site and the site-prediction methods. P Natl Acad Sci USA 2009, 106(16):6700-6705

33. Bahi N, Friocourt G, Carrie A, Graham ME, Weiss JL, Chafey P, Fauchereau F, Burgoyne RD, Chelly J: IL1 receptor accessory protein like, a protein involved in X-linked mental retardation, interacts with Neuronal Calcium Sensor-1 and regulates exocytosis. Hum Mol Genet 2003, 12(12):1415-1425.

34. Permyakov SE, Cherskaya AM, Senin II, Zargarov AA, Shulga-Morskoy SV, Alekseev AM, Zinchenko DV, Lipkin VM, Philippov PP, Uversky VN, Permyakov EA: Effects of mutations in the calcium-binding sites of recoverin on its calcium affinity: evidence for successive filling of the calcium binding sites. Protein Eng 2000, 13(11):783-790.

35. Ermilov AN, Olshevskaya EV, Dizhoor AM: Instead of binding calcium, one of the EF-hand structures in guanylyl cyclase activating protein-2 is required for targeting photoreceptor guanylyl cyclase. J Biol Chem 2001, 276(51):48143-48148

36. Weiergraber $\mathrm{OH}$, Senin II, Zernii EY, Churumova VA, Kovaleva NA, Nazipova AA, Permyakov SE, Permyakov EA, Philippov PP, Granzin J, Koch KW: Tuning of a neuronal calcium sensor. J Biol Chem 2006, 281(49):37594-37602

37. Kabbani N, Negyessy L, Lin R, Goldman-Rakic P, Levenson R: Interaction with neuronal calcium sensor NCS-1 mediates desensitization of the D2 dopamine receptor. J Neurosci 2002, 22(19):8476-8486.

38. Olshevskaya EV, Boikov S, Ermilov A, Krylov D, Hurley JB, Dizhoor AM: Mapping functional domains of the guanylate cyclase regulator protein, GCAP-2. J Biol Chem 1999, 274(16):10823-10832.

39. Li WH, Yang J, Gu X: Expression divergence between duplicate genes Trends Genet 2005, 21(11):602-607.

40. Rastogi S, Liberles DA: Subfunctionalization of duplicated genes as a transition state to neofunctionalization. BMC Evol Biol 2005, 5(1):28.

41. Lemos B, Bettencourt BR, Meiklejohn CD, Hartl DL: Evolution of proteins and gene expression levels are coupled in Drosophila and are independently associated with mRNA abundance, protein length, and number of protein-protein interactions. Mol Biol Evol 2005, 22(5):1345-1354.

42. Belyaeva ES, Vlassova IE, Biyasheva ZM, Kakpakov VT, Richards G, Zhimulev IF: Cytogenetic analysis of the 2B3-4-2B11 region of the $X$ chromosome of Drosophila melanogaster. II. Changes in $20-\mathrm{OH}$ ecdysone puffing caused by genetic defects of puff 2B5. Chromosoma 1981, 84(2):207-219.

43. Gertz EM, Yu YK, Agarwala R, Schaffer AA, Altschul SF: Composition-based statistics and translated nucleotide searches: improving the TBLASTN module of BLAST. BMC Bio/ 2006, 4:41.

44. Tweedie S, Ashburner M, Falls K, Leyland P, McQuilton P, Marygold S, Millburn G, Osumi-Sutherland D, Schroeder A, Seal R, Zhang H: FlyBase: enhancing Drosophila Gene Ontology annotations. Nuc Acids Res 2009, 37 Database: D555-559.

45. Lawson D, Arensburger P, Atkinson P, Besansky NJ, Bruggner RV, Butler R, Campbell KS, Christophides GK, Christley S, Dialynas E, Emmert D, Hammond M, Hill CA, Kennedy RC, Lobo NF, MacCallum MR, Madey G, Megy K, Redmond S, Russo S, Severson DW, Stinson EO, Topalis P, Zdobnov EM, Birney E, Gelbart WM, Kafatos FC, Louis C, Collins FH: VectorBase: a home for invertebrate vectors of human pathogens. NuC Acids Res 2007, , 35 Database: D503-505.

46. Boguski MS, Lowe TM, Tolstoshev CM: dbEST-database for "expressed sequence tags". Nature Genet 1993, 4(4):332-333. 
47. Hubbard T, Andrews D, Caccamo M, Cameron G, Chen Y, Clamp M, Clarke L, Coates G, Cox T, Cunningham F, Curwen V, Cutts T, Down T, Durbin R, Fernandez-Suarez XM, Gilbert J, Hammond M, Herrero J, Hotz H, Howe K, lyer V, Jekosch K, Kahari A, Kasprzyk A, Keefe D, Keenan S, Kokocinsci F, London D, Longden I, McVicker G, et al: Ensembl 2005. Nuc Acids Res 2005, , 33 Database: D447-453.

48. Katoh $\mathrm{K}$, Kuma $\mathrm{K}$, Toh H, Miyata T: MAFFT version 5: improvement in accuracy of multiple sequence alignment. Nuc Acids Res 2005, 33(2):511-518.

49. Maddison WP, Maddison DR: MacClade: Analysis of phylogeny and character evolution. Version 3 Sunderland, Massachusetts: Sinauer Associates 1992.

50. Librado P, Rozas J: DnaSP v5: a software for comprehensive analysis of DNA polymorphism data. Bioinformatics 2009, 25(11):1451-1452.

51. Kumar S, Tamura K, Nei M: MEGA3: Integrated software for Molecular Evolutionary Genetics Analysis and sequence alignment. Brief Bioinform 2004, 5(2):150-163.

52. Takezaki N, Rzhetsky A, Nei M: Phylogenetic test of the molecular clock and linearized trees. Mol Biol Evol 1995, 12(5):823-833.

53. Drummond AJ, Rambaut A: BEAST: Bayesian evolutionary analysis by sampling trees. BMC Evol Biol 2007, 7:214.

54. Tamura K, Subramanian S, Kumar S: Temporal patterns of fruit fly (Drosophila) evolution revealed by mutation clocks. Mol Biol Evol 2004, 21(1):36-44.

55. Hasegawa $M$, Kishino $H$, Yano $T$ : Dating of the human-ape splitting by a molecular clock of mitochondrial DNA. J Mor Evol 1985, 22(2):160-174.

56. Yang Z: PAML 4: phylogenetic analysis by maximum likelihood. Mol Biol Evol 2007, 24(8):1586-1591.

57. Yang Z: Inference of selection from multiple species alignments. Curr Opin Genet Dev 2002, 12(6):688-694.

58. Whelan S, Goldman N: Distributions of Statistics Used for the Comparison of Models of Sequence Evolution in Phylogenetics. Mol Biol Evol 1999, 16(9):1292-1299.

59. Ovcharenko I, Loots GG, Hardison RC, Miller W, Stubbs L: zPicture: dynamic alignment and visualization tool for analyzing conservation profiles. Genome Res 2004, 14(3):472-477.

60. Frazer KA, Pachter L, Poliakov A, Rubin EM, Dubchak I: VISTA: computational tools for comparative genomics. Nuc Acids Res 2004, , 32 Web Server: W273-279.

61. Boffelli D, McAuliffe J, Ovcharenko D, Lewis KD, Ovcharenko I, Pachter L, Rubin EM: Phylogenetic shadowing of primate sequences to find functional regions of the human genome. Science 2003, 299(5611):1391-1394.

62. Siepel A, Bejerano G, Pedersen JS, Hinrichs AS, Hou M, Rosenbloom K, Clawson H, Spieth J, Hillier LW, Richards S, Weinstock GM, Wilson RK, Gibbs RA, Kent WJ, Miller W, Haussler D: Evolutionarily conserved elements in vertebrate, insect, worm, and yeast genomes. Genome Res 2005, 15(8):1034-1050.

63. Rhead B, Karolchik D, Kuhn RM, Hinrichs AS, Zweig AS, Fujita PA, Diekhans M, Smith KE, Rosenbloom KR, Raney BJ, et al: The UCSC genome browser database: update 2010. Nucl Acids Res 2009, , 38 Database: D613-619.

64. Parker SC, Hansen L, Abaan HO, Tullius TD, Margulies EH: Local DNA topography correlates with functional noncoding regions of the human genome. Science 2009, 324(5925):389-392.

65. Matys V, Kel-Margoulis OV, Fricke E, Liebich I, Land S, Barre-Dirrie A, Reuter I, Chekmenev D, Krull M, Hornischer K, Voss N, Stegmaier P, LewickiPotapov B, Saxel H, Kel AE, Wingender E: TRANSFAC and its module TRANSCompel: transcriptional gene regulation in eukaryotes. Nuc Acids Res 2006, , 34 Database: D108-110.

66. Sandelin A, Alkema W, Engstrom P, Wasserman WW, Lenhard B: JASPAR: an open-access database for eukaryotic transcription factor binding profiles. Nuc Acids Res 2004, 32 Database: D91-94.

67. Fares MA, McNally D: CAPS: coevolution analysis using protein sequences. Bioinformatics 2006, 22(22):2821-2822.

68. Gu X: Maximum-likelihood approach for gene family evolution under functional divergence. Mol Biol Evol 2001, 18(4):453-464.

69. GU X, Velden Vander K: DIVERGE: phylogeny-based analysis for functionalstructural divergence of a protein family. Bioinformatics 2002, 18(3):500-501.
70. Guindon S, Gascuel O: A simple, fast, and accurate algorithm to estimate large phylogenies by maximum likelihood. Syst Biol 2003, 52(5):696-704.

71. Posada D, Crandall KA: MODELTEST: testing the model of DNA substitution. Bioinformatics 1998, 14(9):817-818.

72. Kosakovsky Pond SL, Frost SDW, Muse SV: HyPhy: hypothesis testing using phylogenies. Bioinformatics 2005, 21(5):676-679.

73. Arnold K, Bordoli L, Kopp J, Schwede T: The SWISS-MODEL workspace: a web-based environment for protein structure homology modelling. Bioinformatics 2006, 22(2):195-201.

74. Berman HM, Westbrook J, Feng Z, Gilliland G, Bhat TN, Weissig H, Shindyalov IN, Bourne PE: The Protein Data Bank. Nuc Acids Res 2000, 28(1):235-242.

75. Bourne $Y$, Dannenberg J, Pollmann V, Marchot P, Pongs O: Immunocytochemical localization and crystal structure of human Frequenin (neuronal calcium sensor 1). J Biol Chem 2001, 276(15):11949-11955.

76. Guex N, Peitsch MC: SWISS-MODEL and the Swiss-PdbViewer: an environment for comparative protein modeling. Electrophoresis 1997, 18(15):2714-2723.

doi:10.1186/1471-2148-10-54

Cite this article as: Sánchez-Gracia et al:: Two Frequenins in Drosophila: unveiling the evolutionary history of an unusual Neuronal Calcium Sensor (NCS) duplication. BMC Evolutionary Biology 2010 10:54.

\section{Submit your next manuscript to BioMed Central and take full advantage of:}

- Convenient online submission

- Thorough peer review

- No space constraints or color figure charges

- Immediate publication on acceptance

- Inclusion in PubMed, CAS, Scopus and Google Scholar

- Research which is freely available for redistribution

Submit your manuscript at www.biomedcentral.com/submit
C Biomed Central 